\title{
Endoscopic mucosal resection for diagnosis of peripheral T-cell lymphoma arising in the duodenum
}

\author{
Masashi Yamamoto a, Tsutomu Nishidaa ${ }^{\text {, Dai Nakamatsua }}$, Shiro Adachi ${ }^{\mathrm{b}}$
}

Toyonaka Municipal Hospital, Osaka, Japan

A 66-year-old man visited our institution because of upper abdominal pain. Esophagogastroduodenoscopy revealed that the duodenum bulb had become erythematous and rough circumferentially. An irregular ulcer was detected close to the pylorus and many whitish granules were seen near to the superior duodenal angulus. Endoscopic ultrasonography showed that the second layer was prominently thick and homogenously hypoechoic (Fig. 1). First, we performed biopsies that showed some atypical lymphoid cells. However, the biopsy specimens included an insufficient number of atypical lymphoid cells for a definitive diagnosis. Therefore, we performed diagnostic endoscopic mucosal resection (EMR) to obtain larger specimens. EMR was carried out safely, without any adverse event. The EMR specimen revealed infiltrative growth of moderately enlarged atypical lymphoid cells with abundant clear cytoplasm in the interfollicular zone of the mucosal stroma. They occasionally invaded and destroyed the crypt epithelium. They were immunopositive for CD3 and CD4, but negative for CD56 and CD30 (Fig. 2). Finally, we made the definite diagnosis of peripheral T-cell lymphoma (PTCL).

PTCL is a rare entity among malignant lymphomas. The endoscopic appearance of gastrointestinal PTCL is too various and too nonspecific for diagnosis, e.g., ulcerative, ulceroinfiltrative and ulcerofungating [1]. Additionally, malignant lymphoma is often difficult to diagnose by endoscopic biopsy. Iwamuro et al reported that 9 (18.8\%) of 48 patients with gastrointestinal follicular lymphoma were not appropriately diagnosed by initial endoscopic biopsy [2]. We herein report a case of duodenal PTCL which EMR enabled us to diagnose.

Department of a Gastroenterology (Masashi Yamamoto, Tsutomu

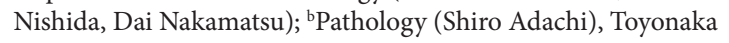
Municipal Hospital, Osaka, Japan

Conflict of Interest: None

Correspondence to: Masashi Yamamoto, M.D., Department of Gastroenterology, Toyonaka Municipal Hospital, 4-14-1 Shibahara, Toyonaka, Osaka 560-8565 Japan, e-mail: jqwty188@ybb.ne.jp

Received 30 November 2018; accepted 15 December 2018; published online 15 January 2019

DOI: https://doi.org/10.20524/aog.2019.0346

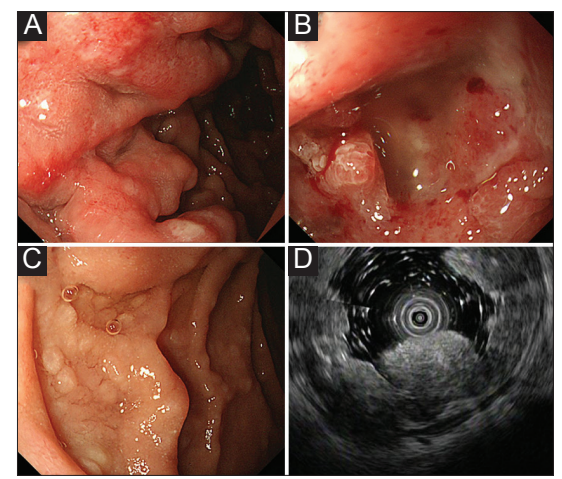

Figure 1 (A) White light endoscopy shows reddish and rough mucosa spreading over the duodenal bulb, (B) irregular ulcer close to the pylorus, and $(C)$ many small white granules crowded near to the superior duodenal angulus. (D) Endoscopic ultrasonography shows a homogenously hypoechoic thickened second layer that expands into the third layer

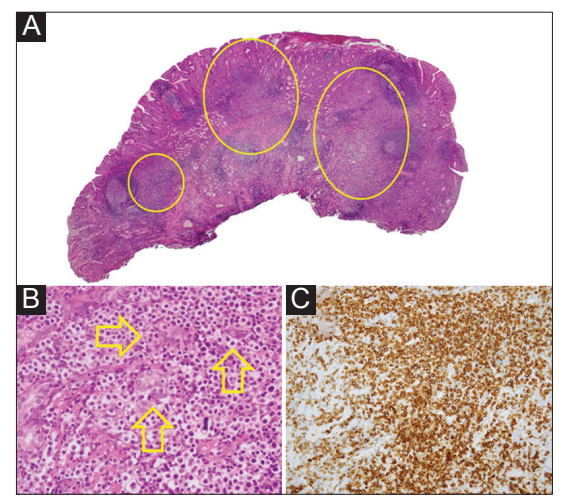

Figure 2 (A) Hematoxylin-eosin staining of the endoscopic mucosal resection specimen showed that expansion of the interfollicular zone and a decreased density of crypts (glands) were observed at the lowest power (circle). (B) The highest power showed that enlarged atypical lymphoid cells (three or more times larger than normal lymphocytes) with abundant clear cytoplasm had infiltrated and invaded the cryptic epithelium (arrow). (C) Immunohistochemical examination revealed that the atypical lymphoid cells expressed CD3

\section{References}

1. Vetro C, Bonanno G, Giulietti G, et al. Rare gastrointestinal lymphomas: the endoscopic investigation. World J Gastrointest Endosc 2015;7:928-949.

2. Iwamuro M, Okada H, Takata $K$, et al. Diagnostic accuracy of endoscopic biopsies for the diagnosis of gastrointestinal follicular lymphoma: a clinicopathologic study of 48 patients. Ann Diagn Pathol 2014;18:99-103. 